\title{
(2) OPEN ACCESS \\ Validation of ICD-10-CM codes for injuries complicating pregnancy, childbirth and the puerperium: a medical record review
}

\author{
Anna Hansen (D) , 1,2,3 Dana Quesinberry, ${ }^{1,4}$ Peter Akpunonu (D) , Julia Martin, ${ }^{5}$ \\ Svetla Slavova (1) 1,6
}

- Additional material is published online only. To view please visit the journal online (http://dx.doi.org/10.1136/ injuryprev-2019-043512).

${ }^{1}$ Kentucky Injury Prevention and Research Center, University of Kentucky, Lexington, Kentucky, USA

${ }^{2}$ Sociology, University of Kentucky, Lexington, Kentucky, USA

${ }^{3}$ College of Medicine, University of Kentucky, Lexington,

Kentucky, USA

${ }^{4}$ Health Management \& Policy, University of Kentucky, Lexington, Kentucky, USA ${ }^{5}$ Department of Emergency Medicine, College of Medicine, University of Kentucky, Lexington, Kentucky, USA ${ }^{6}$ Department of Biostatistics, College of Public Health, University of Kentucky, Lexington, Kentucky, USA

Correspondence to Dr Svetla Slavova, Healthy Kentucky Research Building, Office 261, 760 Press Ave, University of Kentucky, Lexington, KY 40536, USA; ssslav2@email.uky.edu

Received 10 January 2020 Revised 9 September 2020 Accepted 26 September 2020

\section{ABSTRACT \\ Introduction The purpose of this study was to estimate} the positive predictive value (PPV) of International Classification of Diseases, 10th Revision, Clinical Modification (ICD-10-CM) codes for injury, poisoning, physical or sexual assault complicating pregnancy, childbirth and the puerperium (PCP) to capture injury encounters within both hospital and emergency department claims data.

Methods A medical record review was conducted on a sample ( $n=157$ ) of inpatient and emergency department claims from one Kentucky healthcare system from 2015 to 2017, with any diagnosis in the ICD-10-CM range 09A.2-09A.4. Study clinicians reviewed medical records for the sampled cases and used an abstraction form to collect information on documented presence of injury and PCP complications. The study estimated the PPVs and the $95 \%$ Cls of 09A.2-09A.4 codes for (1) capturing injuries and (2) capturing injuries complicating PCP. Results The estimated PPV for the codes 09A.209A.4 to identify injury in the full sample was $79.6 \%$ (95\% Cl 73.3\% to $85.9 \%$ ) and the PPV for capturing injuries complicating PCP was $72.0 \%$ (95\% Cl $65.0 \%$ to $79.0 \%$ ). The estimated PPV for an inpatient principal diagnosis 09A.2-09A.4 to capture injuries was $90.7 \%$ (95\% Cl $82.0 \%$ to $99.4 \%$ ) and the PPV for capturing injuries complicating PCP was $88.4 \%$ (95\% Cl $78.4 \%$ to 98.4\%). The estimated PPV for any mention of 09A.209A.4 in emergency department data to capture injuries was $95.2 \%$ (95\% Cl $90.6 \%$ to $99.9 \%$ ) and the PPV for capturing injuries complicating PCP was $81.0 \%(95 \% \mathrm{Cl}$ $72.4 \%$ to $89.5 \%$ ).

Discussion The 09A.2-09A.4 codes captured high percentage true injury cases among pregnant and puerperal women.

\section{INTRODUCTION}

Traumatic injuries are a prevalent and problematic complication of pregnancy in the USA. Traumatic injuries complicate approximately $8 \%$ of all pregnancies, and are the leading non-obstetric cause of maternal death in the USA. ${ }^{12}$ Pregnancy has also been demonstrated as a risk factor for traumatic injuries and trauma-related mortality. Pregnant women are both more likely to experience certain traumatic injuries (namely, violent traumas) and more likely to die when traumatic injuries occur. ${ }^{3}$ Women who are pregnant at the time of a traumatic injury are at a two-fold higher risk for mortality compared with their non-pregnant counterparts. ${ }^{3}$
Despite the risk of poor injury-related outcomes among pregnant women, research concerning traumatic injuries in pregnant and puerperal patients is limited. This is in part due to shortcomings in the ability to identify pregnant and puerperal patients in morbidity data. Administrative data from hospital and emergency department admissions are primary sources for injury morbidity surveillance used to inform public health interventions. On 1 October 2015, medical coding in the US transitioned from the International Classification of Diseases, Ninth Revision, Clinical Modification (ICD-9-CM) to the 10th Revision (ICD-10-CM). The number of injury diagnosis codes increased from approximately 2600 codes in ICD-9-CM to 43000 codes in ICD-10-CM, ${ }^{4}$ and also included new codes specific to injuries complicating pregnancy, childbirth and the puerperium (PCP) (O9A.2-O9A.5). ${ }^{56}$ In July 2019, the Centers for Disease Control and Prevention (CDC) published an ICD-10-CM surveillance case definition for injury hospitalisations. ${ }^{7}$ This definition included principal diagnoses of O9A.2-O9A.5, injuries complicating pregnancy, childbirth, and the puerperium, in the inclusion criteria. $^{78}$

The ability of the O9A.2-O9A.5 codes to identify true injury cases is unknown. Furthermore, per the ICD-10-CM guidelines for Coding and Reporting, ${ }^{5}$ codes from Chapter 15, PCP (O00-O9A) 'have sequencing priority over codes from other chapters. Additional codes from other chapters may be used in conjunction with Chapter 15 codes to further specify conditions.' Specifically, O9A.2 'should be sequenced first, followed by the appropriate injury, poisoning, toxic effect, adverse effect or underdosing code, and then the additional code(s) that specifies the condition caused by the poisoning, toxic effect, adverse effect or underdosing'. Similarly, O9A.3-O9A.4 should be sequenced first, followed by the appropriate codes to identify any associated current injury due to physical abuse or sexual abuse. However, it has not been studied how often and why the O9A.2-O9A.5 are listed as a secondary diagnosis, and what percentage of injuries complicating pregnancy-related outcomes are potentially missed when the definition for injury hospitalisations captures only principal diagnoses of O9A.2-O9A.5.

ICD-10-CM coding of inpatient and emergency department discharge data follows guidelines for assigning a principal diagnosis. In inpatient data, the 
principal diagnosis is defined as 'that condition established after study to be the chiefly responsible for occasioning the admission of the patient to the hospital for care'. 5 This definition does not apply to hospital-based outpatient services, including emergency department visits. In outpatient settings, the first-listed diagnosis represents 'the code for the diagnosis, condition, problem or other reason for the encounter/visit shown in the medical record as chiefly responsible for the service provided. In some cases the first-listed diagnosis may be a symptom when a diagnosis has not been established (confirmed) by the physician'.5

This analysis evaluates the ability of O9A.2-O9A.5 codes to identify injuries in inpatient and emergency department discharge claims from one healthcare facility in Kentucky, looking additionally at the codes' positive predictive value (PPV) when used as a principal diagnosis within inpatient data, as a secondary diagnosis within inpatient data, and in any position within emergency department data.

The primary goals of this study were (1) to assess how the codes for injury complicating PCP (O9A.2-O9A.5) have been used in inpatient and emergency department billing records; (2) to estimate the ability of these codes to capture true positive cases for injuries (ie, estimate the PPV for this group of codes to capture injury cases); and (3) to estimate the codes' PPV for capturing injuries complicating pregnancy-related outcomes.

\section{METHODS}

The research team conducted a retrospective study using administrative billing records for inpatient treatment and emergency department visits from one healthcare system in Kentucky. All records with discharge dates between 1 October 2015 and 30 December 2017 with a principal (first listed) or a secondary diagnosis code in the ICD-10-CM range O9A.2-O9A.5 were eligible for inclusion. During the study period there were no records with a diagnosis code of O9A.5, indicating treatment episodes of psychological abuse complicating PCP. Therefore, the study's main objective was refocused on estimating the PPV for the group of codes O9A.2-O9A.4. (O9A.2 injury, poisoning and certain other consequences of external causes complicating pregnancy, PCP'; O9A.3 'Physical abuse complicating PCP'; O9A.4 'Sexual abuse complicating PCP'). ${ }^{5}$ A total of 157 records with a diagnosis of O9A.2-O9A.4 in any field were identified, providing no larger than $8 \%$ margin of error for the $95 \%$ $95 \%$ CI of the estimated PPV, under the assumption that the true PPV was $70 \%$.

A medical record review study was designed to determine whether the 157 encounters of care were 'true positive' cases for (1) an injury, and (2) an injury that complicates PCP. A data abstraction form was developed with input from an emergency medicine physician and injury epidemiologists (online supplemental file 1). The form was used to collect information on the documented presence of injury, poisoning, physical or sexual abuse (a.k.a. 'injury' for simplicity of notation), the mechanism and intent of injury, and the presentation of certain signs and symptoms which may indicate obstetric complications (eg, abdominal pain, loss of fluids/excessive vaginal discharge, vaginal bleeding, abnormal fetal movement or heartrate). The specific abstract form questions were: 'Does this encounter of care involve an injury?' and 'Based on the reviewer's assessment, does this encounter of care involve an injury that complicated pregnancy, delivery, or the puerperium?' Abstracted data were collected via Research Electronic Data Capture, a secure, webbased application. Each case was reviewed by two independent reviewers, namely an emergency medicine physician and a medical student. A third reviewer, an emergency medicine physician, adjudicated cases where the two abstractors disagreed.

The PPV of O9A.2-O9A.4 codes for capturing injuries was estimated as the proportion of cases confirmed by the study reviewers as injuries (ie, a positive response to the question 'Does this encounter of care involve an injury?'). The PPV of the O9A.2-O9A.4 codes for capturing injuries complicating pregnancy-related outcomes was estimated by the proportion cases with a positive response to the question 'Based on the reviewer's assessment, does this encounter of care involve an injury that complicated PCP?'

Statistical analysis was done with Stata V.15 and SAS V.9.4. ${ }^{9} 10$ The estimated PPVs were reported with their 95\% CI. The agreements between the two study abstractors (before adjudication) was measured by prevalence-adjusted bias-adjusted kappa (PABAK), ${ }^{11}$ which was determined using an online PABAK calculator. $^{12}$

\section{RESULTS}

The study sample included 157 cases discharged from inpatient $(n=73)$ or emergency department setting $(n=84)$. The majority (93.6\%) of the cases were captured by a code O9A.2 (injury, poisoning and certain other consequences of external causes); $3.8 \%$ by O9A.3 (physical abuse), and $2.6 \%$ by O9A.4 (sexual abuse). Of the 157 records, 114 had principal or first-listed diagnosis code O9A.2-O9A.4, including in 43 (58.9\%) of the inpatient cases. Thirty-seven cases had a principal or first-listed diagnosis of an O-Code other than O9A.2-O9A.4, and six cases had a code from another ICD-10-CM chapter. An external cause of injury ICD-10-CM code was listed in 129 (82.2\%) of the administrative billing records.

The analysis of the abstracted data showed that the two reviewers agreed in their assessment for involvement of injury in 132 of the 157 cases (84.1\% agreement, PABAK 0.68). A third abstractor reviewed the cases of disagreement and the adjudicated dataset was used for the reported results below.

The reviewers found sufficient documentation in the medical records to confirm the existence of an injury in 125 of 157 cases. Therefore, the estimated PPV for codes O9A.2-O9A.4 to identify an injury encounter was $79.6 \%$ (95\% CI $73.3 \%$ to $85.9 \%$; table 1). In 113 of the 157 cases, the abstractors found sufficient documentation for injury accompanied by symptoms of obstetric complications. Thus, the estimated PPV for codes O9A.2-O9A.4 to identify encounters of care involving injury complicating PCP was $72.0 \%$ (95\% CI $65.0 \%$ to $79.0 \%)$.

Among the 43 inpatient cases with a principal diagnosis in the range O9A.2-O9A.4, 39 were confirmed as injuries and 38 were confirmed as injuries complicating PCP. Therefore, the estimated PPV for an inpatient principal diagnosis O9A.2-O9A.4 to capture an injury was $90.7 \%$ (95\% CI $82.0 \%$ to $99.4 \%$ ) and to capture an injury PCP was $88.4 \%$ (95\% CI $78.4 \%$ to 98.4\%). Of the 30 inpatient cases with a secondary diagnosis in the range O9A.2-O9A.4, six were confirmed as injuries and seven were confirmed as injuries complicating PCP (note: there was one complicated encounter that required adjudication and was determined as an encounter not involving care for an injury but involving care for pregnancy complications due to a previous injury/assault). Therefore, the estimated PPV for an inpatient secondary diagnosis O9A.2-O9A.4 to capture an injury was $20.0 \%$ (95\% CI $4.8 \%$ to $35.2 \%$ ) and to capture an injury complicating PCP was $23.3 \%$ (95\% CI $7.3 \%$ to $39.4 \%$ ).

Of the 84 emergency department cases with any diagnosis in the range O9A.2-O9A.4, 80 were confirmed as injuries and 


\begin{tabular}{|c|c|c|}
\hline & $\begin{array}{l}\text { No of } \\
\text { records }\end{array}$ & $\begin{array}{l}\text { Estimated PPV, \% } \\
(95 \% \mathrm{Cl})\end{array}$ \\
\hline \multicolumn{3}{|l|}{ All cases $(n=157)$} \\
\hline Confirmed injury & 125 & 79.6 (73.3 to 85.9$)$ \\
\hline Confirmed injury complicating PCP & 113 & $72.0(65.0$ to 79.0$)$ \\
\hline \multicolumn{3}{|l|}{ Inpatient discharge data $(n=73)$} \\
\hline \multicolumn{3}{|l|}{ Primary diagnosis $(n=43)$} \\
\hline Confirmed injury & 39 & 90.7 (82.0 to 99.4$)$ \\
\hline Confirmed injury complicating PCP & 38 & 88.4 (78.4 to 98.4$)$ \\
\hline \multicolumn{3}{|l|}{ Secondary diagnosis $(n=30)$} \\
\hline Confirmed injury & 6 & 20.0 (4.8 to 35.2$)$ \\
\hline Confirmed injury complicating PCP & 7 & 23.3 (7.3 to 39.4$)$ \\
\hline \multicolumn{3}{|l|}{ Total } \\
\hline Confirmed injury & 45 & $61.6(50.2$ to 73.1$)$ \\
\hline Confirmed injury complicating PCP & 45 & $61.6(50.2$ to 73.1$)$ \\
\hline \multicolumn{3}{|c|}{ Emergency department discharge data $(n=84)$} \\
\hline Confirmed injury & 80 & $95.2(90.6$ to 99.9$)$ \\
\hline Confirmed injury complicating PCP & 68 & $81.0(72.4$ to 89.5$)$ \\
\hline
\end{tabular}

2-09A.4 to capture injuries and injuries complicating PCP, in an electronic health record case confirmation study in a Kentucky healthcare system, October 2015 to December 2017.

ICD-10-CM, International Classification of Diseases, 10th Revision, Clinical Modification; PCP, pregnancy, childbirth and the puerperium.

68 were confirmed as injuries complicating PCP. Therefore, the estimated PPV for an emergency department diagnosis O9A.2-O9A.4 to capture an injury was 95.2\% (95\% CI 90.6\% to $99.9 \%$ ) and to capture an injury complicating PCP was $81.0 \%$ (95\% CI $72.4 \%$ to $89.5 \%$ ).

Among the cases with confirmed injury complicating pregnancy-related outcomes $(n=113)$, the most frequent obstetric complications were abdominal pain/cramping (27.4\%), contractions (4.4\%), vaginal bleeding (4.4\%), abnormal fetal movement $(3.5 \%)$ or maternal death $(3.5 \%)$ (table 2$)$. Also, in the cases confirmed as injuries $(n=125)$, the reviewers identified $68.0 \%$ as unintentional, $15.2 \%$ as assault, $5.6 \%$ as selfinflicted and $11.2 \%$ as undetermined intent (table 3). The most frequently identified injury causes among the confirmed injury cases were motor vehicle crash (37.2\%) and fall (19\%).

Overall, $32(20.4 \%)$ of the cases were not confirmed as injuries. These cases included a diverse range of medical presentations in which an injury event was often not clearly recorded. These cases commonly included minor, non-specific symptoms which were not directly attributed to a reported injury. Other cases were medically and socially complex. For instance, two cases captured delivery hospitalisations for pregnancies resulting from rape. Multiple cases involved suspected adverse medication reactions, surgical wound infections, or sequela of intravenous

Table 2 Signs and symptoms among confirmed cases of injury complicating PCP $(n=113)$

\begin{tabular}{ll}
\hline Signs and symptoms $(\mathbf{n}=113)$ & No of records $(\%)$ \\
\hline Abdominal pain & $31(27.4)$ \\
Contractions & $5(4.4)$ \\
Vaginal bleeding & $5(4.4)$ \\
Abnormal fetal movement & $4(3.5)$ \\
Maternal death & $4(3.5)$ \\
\hline
\end{tabular}

$\mathrm{PCP}$, pregnancy, childbirth and the puerperium
Table 3 Intentions of injury among confirmed injury cases $(n=125)$

\begin{tabular}{ll}
\hline Intentions & No of records $(\%)$ \\
\hline Unintentional & $85(68.0)$ \\
Assault & $19(15.2)$ \\
Self-inflicted & $7(5.6)$ \\
Undetermined intent & $14(11.2)$ \\
\hline
\end{tabular}

drug use. Additionally, several cases involved severe obstetric complications such as pre-eclampsia, placental abruption, postpartum cardiomyopathies and hemolysis, elevated liver enzymes, and low platelet count (HELLP) syndrome.

\section{DISCUSSION}

This study provides strong support for the inclusion of O9A.2-O9A.4 principal diagnosis codes into the ICD-10-CM updated surveillance case definition for injury hospitalisations. The results from this study demonstrated that the set of codes O9A.2-O9A.4 had a high PPV for capturing injuries $(79.6 \%$; $95 \%$ CI $73.3 \%$ to $85.9 \%$ ) as well as injuries complicating PCP (72.0\%; 95\% CI $65.0 \%$ to $79.0 \%)$. The estimated PPV for capturing injuries was even higher when the codes were assigned as principal diagnoses in inpatient records, $(90.7 \%$; $95 \% \mathrm{CI}$ $82.0 \%$ to $99.4 \%)$.

There existed marked overlap between confirmed injury cases and confirmed injuries complicating PCP. The PPV increased minimally when considering either an injury or an injury complicating PCP. Specifically, the estimated PPV for codes O9A.2-O9A.4 to identify encounters of care involving either an injury or an injury complicating PCP was $80.3 \%$ (95\% CI 73.2\% to $86.2 \%)$.

The study findings suggest that medical coders may benefit from continuing education on coding for injuries complicating PCP. For example, in 4\% of the study cases the codes O9A.2-O9A.4 did not have the correct sequencing priority. ${ }^{5}$ The code O9A.2 'should be sequenced first, followed by the appropriate injury, poisoning, toxic effect, adverse effect or underdosing code, and then the additional code(s) that specifies the condition caused by the poisoning, toxic effect, adverse effect or underdosing'. ${ }^{13}$ Similarly, O9A.3-O9A.4 should be sequenced first, followed by the appropriate codes to identify any associated current injury due to physical abuse or sexual abuse.

The medical record review suggested that continuing education could improve the documentation and coding of some medically and socially complicated cases. For example, in cases where the injury occurred early in the pregnancy but did not complicate the pregnancy until delivery, the question of whether to assign an O9A.2-.4 code to this type of cases necessitates inclusion in the physician notes of a clear connection of an earlier injury to the subsequent pregnancy complication. The sole case confirmed as an encounter of care for injury complicating PCP but not confirmed as an encounter of care for injury involved complex circumstances. Specifically, this case captured the delivery hospitalisation of a woman with a term delivery of a pregnancy resulting from a sexual assault. This case represented care for a long-term complication of an injury (ie, the delivery of a term pregnancy), but was not considered an encounter of care for the injury itself (ie, the assault). This nuanced interpretation and temporal relationship between injury and pregnancy complication is particularly important where sexual assault resulted in pregnancy and other cases where prenatal care was severely delayed as a result of the injury. 
A limitation of this study is the relatively small sample size. Another limitation of the study is that the findings are only generalisable to the single hospital system population where the study cases where treated. Additional and larger case confirmation studies are needed to estimate the ability of the group of codes O9A.2-O9A.5 as well as the ability of each individual code to capture true positive cases of injuries as well as injuries complicating PCP in different healthcare settings. These results support the need for additional validation studies that could inform decisions on developing a focused surveillance definition for 'injuries complicating pregnancy, delivery or puerperium' to support research and implementation of future injury prevention strategies targeting this population.

The previously proposed CDC injury surveillance definition $^{6}$ stated that 'the inclusion criteria for an injury hospitalisation requires a case to have a principal diagnosis of one of the included nature-of-injury (injury diagnosis) codes' but the list of included diagnosis codes considered injury codes did not contain the range O9A.2-O9A.5. Due to the specific sequencing requirement for coding of injuries complicating pregnancyrelated outcomes, inpatient cases with a principal diagnosis of O9A.2-O9A.5 would have been previously systematically missed. This study provides evidence that the inclusion of O9A.2-O9A.4 codes in the updated CDC surveillance case definition for injury hospitalisations ${ }^{7}$ is justified and will result in capturing true injury cases among pregnant and puerperal women.

\section{What is already known on the subject}

- Pregnancy is a risk factor for traumatic injuries and traumarelated mortality.

- Improved surveillance is needed concerning injuries in pregnant and puerperal patients.

\section{What this study adds}

- The inclusion of 09A.2-09A.4 codes in the surveillance case definition for injury hospitalisations is justified and will result in capturing true injury cases among pregnant and puerperal women.

Acknowledgements The authors would like to thank the Kentucky Office of Health Data and Analytics and the Kentucky Department for Public Health, Cabinet for Health and Family Services, for providing data and support. The Kentucky Injury Prevention and Research Center is a bona fide agent for the Kentucky Department for Public Health in the area of injury prevention and injury surveillance.

Contributors AH performed data abstractions and contributed to manuscript development. DQ provided methodological oversight and contributed to manuscript development. As emergency medicine physicians, PA and JM guided development of the abstract form and helped with interpreting the clinical relevance of results. SS provided methodological input and oversight on the project, provided statistical support, facilitated collaborations with physician consults, and contributed to all sections of manuscript developement.
Funding This publication was supported by the Cooperative Agreement Number, U17 CE924846, funded by the Centers for Disease Control and Prevention.

Disclaimer Its contents are solely the responsibility of the authors and do not necessarily represent the official views of the Centers for Disease Control and Prevention or the Department of Health and Human Services.

Competing interests None declared.

Patient consent for publication Not required.

Ethics approval This study was approved by the University of Kentucky Institutional Review Board.

Provenance and peer review Commissioned; externally peer reviewed.

Data availability statement No data are available.

Supplemental material This content has been supplied by the author(s). It has not been vetted by BMJ Publishing Group Limited (BMJ) and may not have been peer-reviewed. Any opinions or recommendations discussed are solely those of the author(s) and are not endorsed by BMJ. BMJ disclaims all liability and responsibility arising from any reliance placed on the content. Where the content includes any translated material, BMJ does not warrant the accuracy and reliability of the translations (including but not limited to local regulations, clinical guidelines, terminology, drug names and drug dosages), and is not responsible for any error and/or omissions arising from translation and adaptation or otherwise.

Open access This is an open access article distributed in accordance with the Creative Commons Attribution Non Commercial (CC BY-NC 4.0) license, which permits others to distribute, remix, adapt, build upon this work non-commercially, and license their derivative works on different terms, provided the original work is properly cited, appropriate credit is given, any changes made indicated, and the use is non-commercial. See: http://creativecommons.org/licenses/by-nc/4.0/.

\section{ORCID iDs}

Anna Hansen http://orcid.org/0000-0002-7344-2825

Peter Akpunonu http://orcid.org/0000-0002-6968-686X

Svetla Slavova http://orcid.org/0000-0002-4541-6574

\section{REFERENCES}

1 Huls CK, Detlefs C. Trauma in pregnancy. Semin Perinatol 2018;42:13-20.

2 Mendez-Figueroa H, Dahlke JD, Vrees RA, et al. Trauma in pregnancy: an updated systematic review. Am J Obstet Gynecol 2013;209:1-10.

3 Deshpande NA, Kucirka LM, Smith RN, et al. Pregnant trauma victims experience nearly 2-fold higher mortality compared to their nonpregnant counterparts. Am J Obstet Gynecol 2017;217:590.e1-590.e9.

4 Hedegaard $\mathrm{H}$, Johnson RL, Warner M, et al. Proposed framework for presenting injury data using the International classification of diseases, tenth revision, clinical modification (ICD-10-CM) diagnosis codes. Natl Health Stat Report 2016;89:1-20.

5 PMIC. International classification of diseases 10th revision clinical modification: practice management information Corporation (PMIC) 2016.

6 Hedegaard HB, Johnson RL, Ballesteros MF. Proposed ICD-10-CM surveillance case definitions for injury hospitalizations and emergency department visits. Natl Health Stat Report 2017;100:1-8.

7 Hedegaard H, Johnson R. An updated International classification of Diseases, 10th revision, clinical modification (ICD-10-CM) surveillance case definition for injury hospitalizations. National Health Statistics Reports 2019.

8 Council of State and Territorial Epidemiologists. Preparing your data: nonfatal ED visits (General injury), 2019. Available: https://resources.cste.org//CD-10-CM/Data\% 20Visualization/Preparing\%20Your\%20Data-\%20Nonfatal\%20ED\%20Visits.pdf [Accessed 9 Jan 2020].

9 Stata Statistical Software. Stata version 15. StataCorp LLC. College Station, TX, 2017.

10 SAS. Sas version 9.4. NC: SAS Institute Inc; Cary, 2012.

11 Byrt T, Bishop J, Carlin JB. Bias, prevalence and kappa. J Clin Epidemiol 1993;46:423-9.

12 Olmos A. Kappa online calculator, 2007. Available: http://www.antonio-olmos.com/ kappa/online/calculator.html [Accessed 5 Nov 2019].

13 Center for Medicare and Medicaid Services. ICD-10-CM official guidelines for coding and reporting. 20162019. 